\title{
Testing the magnetic field models of disk galaxies with the SKA
}

\author{
T.G. Arshakian ${ }^{* a}$ R. Stepanov ${ }^{b}$, R. Beck ${ }^{a}$, P. Frick $^{b}$ and M. Krause ${ }^{a}$ \\ ${ }^{a}$ Max-Planck-Institut für Radioastronomie, Auf dem Hügel 69, 53121 Bonn, Germany \\ ${ }^{b}$ Institute of Continuous Media Mechanics, Korolyov str. 1, 614061 Perm, Russia \\ E-mail: tigarempifr-bonn.mpg.de, rodioneicmm.ru, \\ rbeck@mpifr-bonn.mpg.de, frick@icmm.ru, mkrause@mpifr-bonn.mpg.de
}

The future new-generation radio telescope SKA (Square Kilometre Array) and its precursors will provide a rapidly growing number of polarized radio sources. Hundred and thousands polarized background sources can be measured towards nearby galaxies thus allowing their detailed magnetic field mapping by means of Faraday rotation measures (RM). We aim to estimate the required density of the background polarized sources detected with the SKA for reliable recognition and reconstruction of the magnetic field structure in nearby spiral galaxies. We construct a galaxy model which includes the ionized gas and magnetic field patterns of different azimuthal symmetry (axisymmetric (ASS), bisymmetric (BSS) and quadrisymmetric spiral (QSS), and superpositions) plus a halo magnetic field. RM fluctuations with a Kolmogorov spectrum due to turbulent fields and/or fluctuations in ionized gas density are superimposed. Recognition of magnetic structures is possible from RM towards background sources behind galaxies or a continuous RM map obtained from the diffuse polarized emission from the galaxy itself. Under favourite conditions, about a few dozens of polarized sources are sufficient for a reliable recognition. $R e$ construction of the field structure without precognition becomes possible for a large number of background sources. A reliable reconstruction of the field structure needs at least $20 \mathrm{RM}$ values on a cut along the projected minor axis which translates to $\approx 1200$ sources towards the galaxy. Radio telescopes operating at low frequencies (LOFAR, ASKAP and the low-frequency SKA array) may also be useful instruments for field recognition or reconstruction with the help of RM, if background sources are still significantly polarized at low frequencies. This work is a part of the science simulations of cosmic magnetism in the frame of the SKADS (SKA Design Studies).

From Planets to Dark Energy: the Modern Radio Universe

October 1-5 2007

The University of Manchester, UK

\footnotetext{
* Speaker.
} 


\section{Introduction}

Faraday rotation measures $(\mathrm{RM})$ in galaxies are generated by regular fields of the galaxy plus its ionized gas, both of which extend to large galactic radii (Fig. 1). RM towards polarized background sources can trace regular magnetic fields in these galaxies out to even larger distances, however, with the sensitivity of present-day radio telescopes, the number density of polarized background sources is only a few sources per solid angle of a square degree, so that only M 31 and the LMC could be investigated so far $[1,2]$.

Future high-sensitivity radio facilities will observe polarized intensity and RM for a huge number of faint radio sources, thus providing the high density background of polarized point sources. This opens the possibility to study in detail the large-scale patterns of magnetic fields and their superpositions thus allowing the dynamo theory for field amplification and its ordering to be tested. A major step towards a better understanding of galactic magnetism will be achieved by the Square Kilometre Array (SKA, www.skatelescope.org) and its pathfinders.

\section{Models of RM towards foreground galaxy}

The observed RM is a combination of an intrinsic RM of the background sources plus an RM imposed by the foreground galaxy. The model of observed Faraday rotations includes models of the random and regular magnetic fields of the galaxy, the distribution of electron density and the density of polarized background sources [3]. The contribution of the Galactic RM can be accounted properly from the density of RM detected with the LOFAR (Low Frequency Array) or the SKA in a slightly larger region surrounding each galaxy. The intrinsic RM of the background sources will average out if the number of background sources used for the analysis is large enough.

Models for the regular magnetic field in the disk. Although the physics of dynamo action still faces theoretical problems [4], the dynamo is the only known mechanism which is able to generate largescale coherent (regular) magnetic fields of spiral shape. These coherent fields can be represented as a superposition (spectrum) of modes with different azimuthal symmetries. In a smooth, axisymmetric gas disk the strongest mode is that with the azimuthal mode number $m=0$ (ASS spiral field is observed in many nearby galaxies [5, 2]; M 31 in Fig. 1), followed by the weaker $m=1$ mode (BSS spiral fields are rare [6]), and $m=2$ (QSS) [7]. The two magnetic arms (located between the optical spiral arms) in NGC 6946 [8], with the field directed towards the galaxy's centre in both, is a signature of superposed $m=0$ and $m=2$ modes. However, for many of the nearby galaxies for which multi-frequency observations are available, the angular resolutions and/or the signal-tonoise ratios are still insufficient to reveal the dominating magnetic modes or their superpositions. We restrict our analysis to the three lowest azimuthal modes of the toroidal regular magnetic field: ASS, $(m=0)$, BSS spiral $(m=1)$, QSS spiral $(m=2)$, and the superpositions ASS+BSS and ASS+QSS. All modes are assumed to be symmetric with respect to the disk plane (S-modes). The maximum regular field strength is assumed to be $5 \mu \mathrm{G}$ for all modes, consistent with typical values from observations [6].

Models for the regular magnetic field in the halo. Strong vertical fields (so-called X-shaped magnetic fields) extending into the halo are recently observed in several edge-on galaxies such as e.g. M 104, NGC 253, NGC 891 and NGC 5775 [11, 9, 12, 10]. 


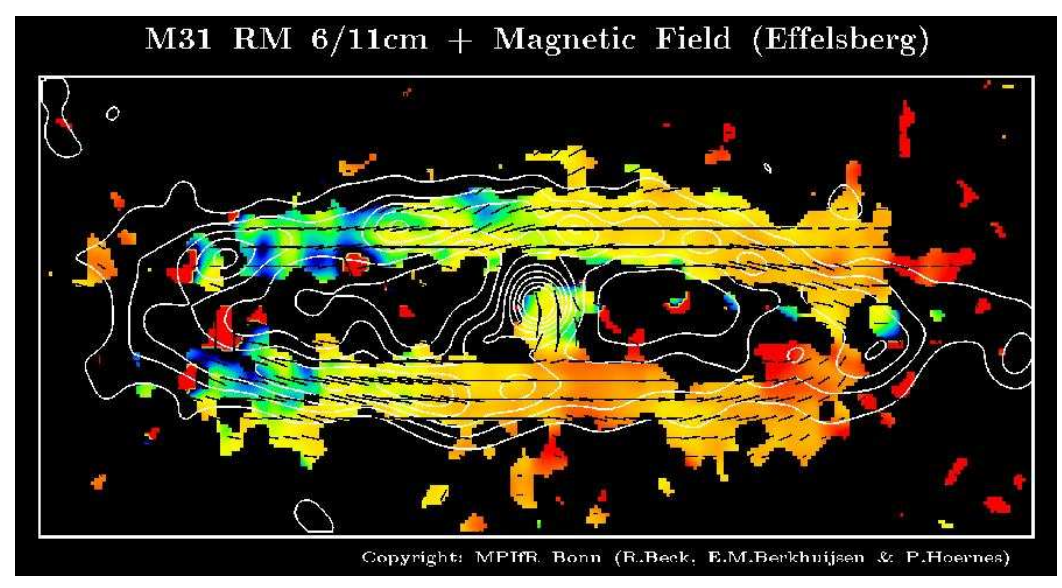

Figure 1: M 31: total emission (contours) and polarized emission (B-vectors) at $\lambda 6.2 \mathrm{~cm}$ superimposed with the distribution of the Faraday rotation measure from diffuse radio emission [5].

Thermal electron density models. We adopt a Gaussian dependence of the electron density on galactic radius $r$ (in the galactic plane) and on height $z$ above the disk midplane. The electron density near the galaxy center, its radial scalelength and vertical scalelength are taken to be $n_{0}=0.03 \mathrm{~cm}^{-3}$, $r_{0}=10 \mathrm{kpc}$ and $h=1 \mathrm{kpc}$, respectively.

Faraday rotation models. The galactic magnetic field is modeled as a superposition of a regular part with a simple azimuthal symmetry $B_{m}$ and a random part $B_{t u r b}$ which describes the contribution of large-scale galactic turbulence. The regular field in the disk is parameterized by pitch angle and the intensity of the corresponding azimuthal mode. The vertical field in the halo increases with height and its direction is tilted at $45^{\circ}$ with respect to the plane, as indicated by the magnetic field structure observed in the halo of nearby galaxies. The Faraday rotation is then modeled as the product of electron density and magnetic field strength integrated along the line of sight (see Fig. 2).

The number density of polarized background sources is simulated using the number counts of polarized sources at $1.4 \mathrm{GHz}[13]$ extrapolated to a polarized flux density limit $(\sim 0.01 \mu \mathrm{Jy})$ reachable with the SKA.

\section{Recognition and reconstruction of regular field structures}

To recognize the large-scale regular component of the magnetic field with a simple symmetry we apply the $\chi^{2}$ minimization between the 'template' and the 'observed' RM patterns for ASS, BSS, and QSS modes, or their superpositions. This method is also used to evaluate the reliability of different modes. Recognition of single and mixed modes of magnetic fields can be reliably performed from a limited sample of RM measurements $(\geq 20)$ towards polarized background sources. Higher modes (BSS and QSS) are easier to recognize, i.e. they need less RM points, shorter observation time and is less affected by the turbulent component. The dependence on turbulence becomes dramatic for weakly inclined (almost face-on) galaxies - a reliable fitting requires a huge number of sources.

When the field structure is more complicated or the turbulent field is stronger then the regular field, the reconstruction method should be applied. This does not need a "precognition" template, 

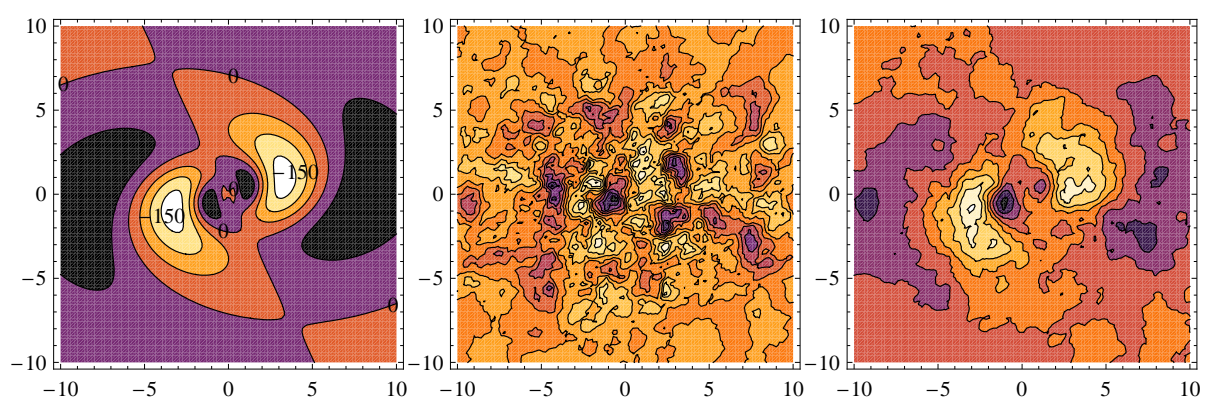

Figure 2: 'Template' RM map (in $\mathrm{rad} \mathrm{m}^{-2}$ ) for an inclination angle of $i=10^{\circ}$ generated for a pure BSS field pattern, for $B=5 \mu \mathrm{G}$ and $n_{0}=0.03 \mathrm{~cm}^{-3}$ (left), a random turbulent field with the r.m.s. level of $30 \mathrm{rad}$ $\mathrm{m}^{-2}$ (middle) and their superposition, the 'observed' RM map (right).

but needs a higher density of RM sources and hence deeper observations [3]. To assess the number of RM points required for a reliable reconstruction we compare the modeled and reconstructed magnetic field profiles of the BSS-type galaxy. The reconstruction method is superior for strongly inclined galaxies (about $70^{\circ}$ and more) and is successful if at least 20 sources are available for one cut along the minor galactic axis (or $\geq 1200$ sources within the solid angle covered by the galaxy).

\section{Perspectives for the SKA}

Recognition or reconstruction of regular field structures from RM data of polarized background sources is a powerful tool for future radio telescopes. Measuring RM at frequencies around $1 \mathrm{GHz}$ with the SKA, simple field structures can be recognized in galaxies up to about $100 \mathrm{Mpc}$ distance and will allow to test dynamo against primordial or other models of field origin for $\approx 60000$ disk galaxies. For this the sensitivity of $0.015 \mu \mathrm{Jy}$ is needed, which can be achieved within $100 \mathrm{~h}$ observation time with the SKA. On the other hand, the reconstruction of magnetic field structures of strongly inclined spiral galaxies would require a sensitivity of the SKA at $1.4 \mathrm{GHz}$ of $\approx 0.5-5 \mu \mathrm{Jy}$ (or an integration time less than one hour) for galaxies at distances of about one Mpc. The field structures of $\approx 60$ galaxies at about $10 \mathrm{Mpc}$ distance can be reconstructed with tens to hundred hours of integration time.

A better knowledge of the slope of number counts $\gamma$ of polarized sources in the flux range accessible to the SKA is crucial for observations of distant galaxies and of the required polarization purity. Observing at larger distances $D$ requires to increase the observing time $T$ according to $T \propto D^{4 / \gamma}$ in order to obtain the same number of sources per solid angle of a galaxy. The recognition method raises requirements to the dynamic range of the telescope for polarization measurements (polarization purity), which may exceed $30 \mathrm{~dB}$ in the case of a flat slope of the number density counts of polarized sources.

Depolarization effects within the background sources, in the foreground of the galaxy or the Milky Way (in the case if the angular extent of a source is larger than the angular turbulence scale in the foreground medium) are yet uncertain. Both depolarization effects, internal and external, depend on the angular resolution and the observing frequency. No statistical data are available yet. 
Future investigations in this direction may modify the number of sources required for a reliable recognition.

As the RM errors are smaller at larger wavelengths the low-frequency SKA array and lowfrequency precursor telescopes like LOFAR would be an ideal tool to study the weak magnetic fields in galaxies and intergalactic space if background sources are still significantly polarized at low frequencies.

\section{References}

[1] J. L. Han, R. Beck, and E. M. Berkhuijsen, New clues to the magnetic field structure of M 31, A\&A 335, 1117-1123 (1998) [arXiv:astro-ph/9805023].

[2] B. M. Gaensler, M. Haverkorn, L. Staveley-Smith, J. M. Dickey, N. M. McClure-Griffiths, J. R. Dickel, and M. Wolleben, The Magnetic Field of the Large Magellanic Cloud Revealed Through Faraday Rotation, Science 307, 1610-1612 (2005) [arXiv: astro-ph/0503226].

[3] R. Stepanov, T. G. Arshakian, R. Beck, P. Frick, and M. Krause, Magnetic field structures of galaxies derived from analysis of Faraday rotation measures, and perspectives for the SKA, ArXiv e-prints 711 (2007) [0711.1267].

[4] A. Brandenburg and K. Subramanian, Astrophysical magnetic fields and nonlinear dynamo theory, Phys. Rep. 417, 1-4 (2005) [arXiv: astro-ph/ 0405052 ].

[5] E. M. Berkhuijsen, R. Beck, and P. Hoernes, The polarized disk in M 31 at $\lambda 6 \mathrm{~cm}$, A\&A 398, 937-948 (2003).

[6] R. Beck, Magnetic Fields in Galaxies in proceedings of Cosmic Magnetic Fields, (R. Wielebinski and R. Beck, eds.), Lecture Notes in Physics, Berlin Springer Verlag, vol. 664, 2005, pp. 41-68.

[7] D. Elstner, R. Meinel, and R. Beck, Galactic dynamos and their radio signatures, A\&AS 94, 587-600 (1992).

[8] R. Beck, Magnetism in the spiral galaxy NGC 6946: magnetic arms, depolarization rings, dynamo modes, and helical fields, A\&A 470, 539-556 (2007) [arXiv:0705.4163].

[9] V. Heesen, M. Krause, R. Beck, and R.-J. Dettmar, The Radio Halo of the Starburst Galaxy NGC 253 in proceedings of The Magnetized Plasma in Galaxy Evolution, (K. T. Chyzy, K. Otmianowska-Mazur, M. Soida, and R.-J. Dettmar, eds.), June 2005, pp. 156-161.

[10] M. Soida, Can we observe the poloidal magnetic field? in proceedings of The Magnetized Plasma in Galaxy Evolution, (K. T. Chyzy, K. Otmianowska-Mazur, M. Soida, and R.-J. Dettmar, eds.), June 2005, pp. 185-190.

[11] M. Krause, R. Wielebinski, and M. Dumke, Radio polarization and sub-millimeter observations of the Sombrero galaxy (NGC 4594). Large-scale magnetic field configuration and dust emission, A\&A 448, 133-142 (2006) [arXiv: astro-ph/0510796].

[12] M. Krause, Large-scale magnetic field in spiral galaxies, Memorie della Societa Astronomica Italiana 78, 314-316 (2007).

[13] A. R. Taylor, J. M. Stil, J. K. Grant, T. L. Landecker, R. Kothes, R. I. Reid, A. D. Gray, D. Scott, P. G. Martin, A. I. Boothroyd, G. Joncas, F. J. Lockman, J. English, A. Sajina, and J. R. Bond, Radio Polarimetry of the ELAIS N1 Field: Polarized Compact Sources, ApJ 666, 201-211 (2007) [arXiv:0705.2736]. 